\title{
Anisotropic Magnification Distortion of the 3D Galaxy Correlation: II. Fourier and Redshift Space
}

\author{
Lam Hui ${ }^{1,2,3}$, Enrique Gaztañaga ${ }^{4}$ and Marilena LoVerde ${ }^{1,2}$ \\ ${ }^{1}$ Institute for Strings, Cosmology and Astroparticle Physics (ISCAP) \\ ${ }^{2}$ Department of Physics, Columbia University, New York, NY 10027, U.S.A. \\ ${ }^{3}$ Institute of Theoretical Physics, The Chinese University of Hong Kong, Hong Kong \\ ${ }^{4}$ Institut de Ciències de l'Espai, IEEC-CSIC, Campus UAB, \\ F. de Ciències, Torre C5 par-2, Barcelona 08193, Spain \\ lhui@astro.columbia.edu,gazta@aliga.ieec.uab.es, marilena@phys.columbia.edu
}

(Dated: October 27, 2018)

\begin{abstract}
In paper I of this series we discuss how magnification bias distorts the $3 \mathrm{D}$ correlation function by enhancing the observed correlation in the line-of-sight (LOS) orientation, especially on large scales. This lensing anisotropy is distinctive, making it possible to separately measure the galaxy-galaxy, galaxy-magnification and magnification-magnification correlations. Here we extend the discussion to the power spectrum and also to redshift space. In real space, pairs oriented close to the LOS direction are not protected against nonlinearity even if the pair separation is large; this is because nonlinear fluctuations can enter through gravitational lensing at a small transverse separation (or i.e. impact parameter). The situation in Fourier space is different: by focusing on a small wavenumber $k$, as is usually done, linearity is guaranteed because both the LOS and transverse wavenumbers must be small. This is why magnification distortion of the galaxy correlation appears less severe in Fourier space. Nonetheless, the effect is non-negligible, especially for the transverse Fourier modes, and should be taken into account in interpreting precision measurements of the galaxy power spectrum, for instance those that focus on the baryon oscillations. The lensing induced anisotropy of the power spectrum has a shape that is distinct from the more well known redshift space anisotropies due to peculiar motions and the Alcock-Paczynski effect. The lensing anisotropy is highly localized in Fourier space while redshift space distortions are more spread out. This means that one could separate the magnification bias component in real observations, implying that potentially it is possible to perform a gravitational lensing measurement without measuring galaxy shapes.
\end{abstract}

PACS numbers: 98.80.-k; 98.80.Es; 98.65.Dx; 95.35.+d

\section{INTRODUCTION}

The effect of magnification bias on the 3D galaxy/quasar correlation function was studied in paper I [1]. (Galaxy and quasar can be considered synonymous hereafter.) With the important exception of the classic paper by Matsubara [2], previous work on how magnification bias modifies clustering observations has largely focused on the 2D angular correlation function [3, 4, 5, 6, 7, 8, 9, 10, 11, 12, 13. The novelty of the 3D correlation function, as emphasized by [2] and [1], is that magnification bias makes it anisotropic. In this paper, we extend our previous analysis by studying the anisotropy in Fourier and redshift space. At first sight, the extension to Fourier space might seem a trivial exercise. The calculations are indeed straightforward, but as we will see, the results are far from obvious: there are important qualitative differences between the results in Fourier space and real space that go beyond the usual wavenumber-position $(k-x)$ duality.

Let us recall the situation in real space, as depicted in paper I. The anisotropy of the observed 3D correlation function can be understood intuitively as follows. The correlation function is measured by pair counts of galaxies. A pair of galaxies that are aligned along the line-of-sight (LOS) behave differently from a pair ori- ented transverse to the LOS. In the former case, the closer galaxy can lens the background one. The same does not happen in the transverse orientation. The net effect is an anisotropy in the observed correlation function, induced by gravitational lensing (or equivalently, magnification bias; we refer to this effect as magnification distortion). This reasoning suggests the gravitational lensing corrections are largest for a pair of galaxies oriented along the LOS. Indeed, the corrections can be quite significant: consider for instance a LOS separation of $\sim 100 \mathrm{Mpc} / \mathrm{h}$; the intrinsic galaxy correlation is rather weak on such a large scale, but the lensing induced correction can be quite substantial, since for the LOS orientation, the relevant lensing impact parameter, i.e. transverse separation, is zero (keep in mind also that the lensing effect grows with the LOS separation while the intrinsic galaxy correlation generally drops with separation). In other words, for the LOS orientation, scales that otherwise would be considered linear can in fact be secretly affected by nonlinear fluctuations via lensing. A large separation $|\delta \mathbf{x}|=\sqrt{\delta \chi^{2}+\left|\delta \mathbf{x}_{\perp}\right|^{2}}$ does not guarantee linearity because nonlinear fluctuations can sneak in through lensing with a small transverse separation $\left|\delta \mathbf{x}_{\perp}\right|$.

This peculiar mixing of linear intrinsic galaxy fluctuations with nonlinear lensing fluctuations does not arise in Fourier space. A small net wavenumber $|\mathbf{k}|$ is sufficient 
to guarantee that both the LOS component $k_{\|}$and the transverse component $\left|\mathbf{k}_{\perp}\right|$ are small. Nonlinear lensing corrections cannot sneak in as long as one focuses on a small $|\mathbf{k}|$, as is usually done. This immediately tells us that the anisotropy of the observed galaxy correlation must appear milder in Fourier space. Our primary goal here is to quantify this.

As discussed in paper I, the magnification bias induced anisotropy in the observed 3D galaxy correlation has two implications. First, precision measurements of the galaxy correlation must take into account such magnification distortion. These include future galaxy surveys that hope to determine the baryon oscillation scale to high accuracy [14, 15, 16, 17, 18, 19, 20]. Second, the distinctive anisotropy pattern makes it possible in principle to separately measure the galaxy-galaxy, galaxy-magnification and magnification-magnification correlations. (The last correlation was generally ignored in previous papers that focused on angular correlation between galaxies at widely separated redshifts $[8,19,10,11,12,13]$, where the galaxymagnification correlation dominates.) Achieving such a separation requires that one understands other sources of anisotropy. We therefore extend our Fourier analysis here to incorporate the anisotropy due to both peculiar motions and the Alcock-Paczynski effect.

The rest of the paper is organized as follows. In $₫ \mathrm{II}$, we derive and numerically compute the magnification distortion of the observed galaxy clustering - \$IA summarizes the results from paper I on the correlation function while \IB focuses on the power spectrum. Redshift space distortion due to peculiar motion is next incorporated in \III and we conclude in $\S \mathrm{IV}$ In Appendix A, we discuss the Alcock-Paczynski anisotropy.

Before we start, it is useful to point out several related papers. Vallinoto et al. 21] explored the impact of lensing, especially magnification bias, on the baryon oscillation signal in the real space correlation function. Their results are consistent with ours in paper I, though they focus exclusively on pair separations that are oriented transverse to the LOS, and their work is therefore more connected to our paper on the angular correlation function 22]. Wagner et al. 23] examined the anisotropy of the 3D correlation that is introduced by light cone effects. A discussion of the classic paper by Matsubara 2] can be found in paper I. Both [2] and paper I focused on the real/configuration space correlation function, though peculiar motions and the Alcock-Paczynski effect are also treated in 2]. A recent paper by Zhang \& Chen 24] explored the effects of gravitational lensing in Fourier space in the context of supernova observations. LoVerde et al. [25] examined the impact of magnification bias on integrated Sachs-Wolfe measurements.

\section{MAGNIFICATION DISTORTION}

Given an intrinsic galaxy overdensity $\delta_{g}$, magnification bias introduces a correction $\delta_{\mu}$ to the observed galaxy overdensity $\delta_{\text {obs }}$ :

$$
\delta_{\mathrm{obs}}=\delta_{g}+\delta_{\mu}
$$

which is a function of the galaxy position, specified for instance by the radial comoving distance $\chi$ and the angular position $\boldsymbol{\theta}$. The magnification bias correction is given by $[\underline{3}, 4,4,[5,[6,6], 8]$ :

$$
\delta_{\mu}=(5 s-2) \kappa
$$

where $\kappa$ is the lensing convergence:

$$
\kappa(\chi, \boldsymbol{\theta})=\int_{0}^{\chi} d \chi^{\prime} \frac{\chi^{\prime}\left(\chi-\chi^{\prime}\right)}{\chi} \nabla_{\perp}^{2} \phi\left(\chi^{\prime}, \boldsymbol{\theta}\right)
$$

$\phi$ is the gravitational potential, and $\nabla_{\perp}^{2}$ is the 2D Laplacian in the transverse directions. We assume a flat universe - generalization to an open or a closed universe is straightforward. The symbol $s$ stands for

$$
s=\frac{d \log _{10} N(<m)}{d m}
$$

where $N(<m)$ is the cumulative number counts for galaxies brighter than magnitude $m$. This assumes the galaxy sample is defined by a sharp faint-end cut-off. A broader definition of $s$ for a more general galaxy selection is given in Appendix A of paper I.

We define the galaxy bias $b$ by $\delta_{g}=b \delta$, where $\delta$ is the mass overdensity. Eq. (11) can then be rewritten as

$$
\frac{\delta_{\text {obs }}}{b}=\delta+\frac{5 s-2}{b} \kappa
$$

The relative importance of the intrinsic clustering and the magnification bias correction is therefore controlled by, among other things, the sample dependent ratio $(5 s-$ 2)/b.

The precise values of $s$ and $b$ depend sensitively on details of how the galaxy/quasar sample is selected, for instance subject to color cuts and so on. Unless otherwise stated, we adopt throughout this paper the value $(5 s-$ $2) / b=1$ to illustrate the effect of magnification bias on clustering measurements (see paper I for more details). An implicit assumption is the linearity of the galaxy bias, a subject we will return to in $₫ \mathrm{IV}$

In all illustrative examples below, we employ the following cosmological parameters: the Hubble constant $h=0.7$, matter density $\Omega_{m}=0.27$, cosmological constant $\Omega_{\Lambda}=0.73$, baryon density $\Omega_{b}=0.046$, power spectrum slope $n=0.95$ and normalization $\sigma_{8}=0.8$. We employ the transfer function of 26], and the prescription of 27] for the nonlinear power spectrum. In all equations we use units where the speed of light is unity: $c=1$.

\section{A. The Correlation Function}

Here, we summarize the main results of paper I. Including lensing magnification, the observed two-point correlation function is given by:

$$
\xi_{\mathrm{obs}}\left(\chi_{1}, \boldsymbol{\theta}_{1} ; \chi_{2}, \boldsymbol{\theta}_{2}\right)=\left\langle\delta_{\mathrm{obs}}\left(\chi_{1}, \boldsymbol{\theta}_{1}\right) \delta_{\mathrm{obs}}\left(\chi_{2}, \boldsymbol{\theta}_{2}\right)\right\rangle
$$




$$
\begin{aligned}
& =\xi_{g g}\left(\chi_{1}, \boldsymbol{\theta}_{1} ; \chi_{2}, \boldsymbol{\theta}_{2}\right)+\xi_{g \mu}\left(\chi_{1}, \boldsymbol{\theta}_{1} ; \chi_{2}, \boldsymbol{\theta}_{2}\right) \\
& +\xi_{g \mu}\left(\chi_{2}, \boldsymbol{\theta}_{2} ; \chi_{1}, \boldsymbol{\theta}_{1}\right)+\xi_{\mu \mu}\left(\chi_{1}, \boldsymbol{\theta}_{1} ; \chi_{2}, \boldsymbol{\theta}_{2}\right)
\end{aligned}
$$

where the magnification bias corrections, the galaxymagnification and magnification-magnification correlations, are:

$$
\begin{array}{r}
\xi_{g \mu}\left(\chi_{1}, \boldsymbol{\theta}_{1} ; \chi_{2}, \boldsymbol{\theta}_{2}\right)+\xi_{g \mu}\left(\chi_{2}, \boldsymbol{\theta}_{2} ; \chi_{1}, \boldsymbol{\theta}_{1}\right)= \\
\frac{3}{2} H_{0}^{2} \Omega_{m}(5 s-2)(1+\bar{z})\left|\chi_{2}-\chi_{1}\right| \\
\int \frac{d^{2} k_{\perp}}{(2 \pi)^{2}} P_{g m}\left(\bar{z}, k_{\perp}\right) e^{i \mathbf{k}_{\perp} \cdot \bar{\chi}\left(\boldsymbol{\theta}_{1}-\boldsymbol{\theta}_{2}\right)} \\
\xi_{\mu \mu}\left(\chi_{1}, \boldsymbol{\theta}_{1} ; \chi_{2}, \boldsymbol{\theta}_{2}\right)=\left[\frac{3}{2} H_{0}^{2} \Omega_{m}(5 s-2)\right]^{2} \\
\int_{0}^{\bar{\chi}} d \chi^{\prime}\left[\frac{\left(\bar{\chi}-\chi^{\prime}\right) \chi^{\prime}}{\bar{\chi}}\right]^{2}\left(1+z^{\prime}\right)^{2} \\
\int \frac{d^{2} k_{\perp}}{(2 \pi)^{2}} P_{m m}\left(z^{\prime}, k_{\perp}\right) e^{i \mathbf{k}_{\perp} \cdot \chi^{\prime}\left(\boldsymbol{\theta}_{1}-\boldsymbol{\theta}_{2}\right)}
\end{array}
$$

Here we have Taylor expanded $\chi_{1}$ and $\chi_{2}$ around the mean $\bar{\chi}$ and retained the lowest order contributions. The intrinsic (unlensed) galaxy auto-correlation, or galaxygalaxy correlation, is

$$
\begin{aligned}
& \xi_{g g}\left(\chi_{1}, \boldsymbol{\theta}_{1} ; \chi_{2}, \boldsymbol{\theta}_{2}\right)= \\
& \xi_{g g}\left(\sqrt{\left(\chi_{1}-\chi_{2}\right)^{2}+\bar{\chi}^{2}\left(\boldsymbol{\theta}_{1}-\boldsymbol{\theta}_{2}\right)^{2}}\right)= \\
& \int \frac{d^{3} k}{(2 \pi)^{3}} P_{g g}(\bar{z}, k) e^{i \mathbf{k} \cdot\left(\mathbf{x}_{1}-\mathbf{x}_{\mathbf{2}}\right)}
\end{aligned}
$$

ignoring for now the issue of redshift distortion, which will be addressed in $₫ \amalg I I$ Note that $\mathbf{x}_{1}$ and $\mathbf{x}_{\mathbf{2}}$ refer to the points corresponding to $\chi_{1}, \boldsymbol{\theta}_{1}$ and $\chi_{2}, \boldsymbol{\theta}_{2}$. Note also $P_{m m}, P_{g m}$ and $P_{g g}$ denote respectively the mass-mass, galaxy-mass and galaxy-galaxy power spectra.

The observed correlation function is a sum of all three correlations above (eq. 7, 8, and 9]). (A discussion of their higher order Taylor corrections can be found in Appendix B of paper I.). Viewed in this way, the anisotropy of the lensing induced corrections is quite striking: $\xi_{g \mu}(1,2)+\xi_{g \mu}(2,1)$ scales linearly with the lineof-sight (LOS) separation $\left|\chi_{2}-\chi_{1}\right|$ (i.e. it increases rather than decreases with the separation!), and $\xi_{\mu \mu}$ is independent of the LOS separation. The intrinsic galaxy autocorrelation $\xi_{g g}$ is isotropic and generally decreases with separation.

We can summarize the distinctive lensing induced anisotropy in the observed correlation function as follows:

$$
\begin{aligned}
\xi_{\text {obs }}\left(\delta \chi, \delta x_{\perp}\right)= & \xi_{g g}\left(\sqrt{\delta \chi^{2}+\delta x_{\perp}^{2}}\right) \\
& +f\left(\delta x_{\perp}\right) \delta \chi+g\left(\delta x_{\perp}\right)
\end{aligned}
$$

where $\delta \chi$ and $\delta x_{\perp}$ are the LOS and transverse separations respectively, $f \delta \chi$ represents the galaxymagnification correlation and $g$ represents the magnification-magnification correlation. Here, $f$ and $g$ are functions of the transverse separation only, and are determined by the galaxy-mass and mass-mass power spectra. This distinctive form of the anisotropy allows us in principle to separately measure $\xi_{g g}, f$ and $g$, from which we can infer the galaxy-galaxy, galaxy-mass and mass-mass power spectra. For instance, at any given $\delta x_{\perp}$, plotting $\xi_{\text {obs }}$ as a function of the LOS separation $\delta \chi$ would reveal a linear contribution at sufficiently large $\delta \chi$ 's where $\xi_{g g}$ is very small. Its slope tells us $f$ and its extrapolation to $\delta \chi=0$ tells us $g$. Subtracting $\delta \chi f+g$ from $\xi_{\text {obs }}$ then yields $\xi_{g g}$. This is illustrated in Fig. 2 of paper I, where we also present order of magnitude estimates for the ratios $\xi_{\mu \mu} / \xi_{g g}$ and $\xi_{g \mu} / \xi_{g g}$ :

$$
\begin{gathered}
\frac{\xi_{\mu \mu}}{\xi_{g g}} \sim\left[\frac{5 s-2}{b}\right]^{2} \frac{(1+\bar{z})^{2}}{50}\left(\bar{\chi} H_{0}\right)^{3} \frac{\pi H_{0}}{k_{*}} \frac{\Delta^{2}\left(k_{*}\right)}{\Delta^{2}\left(k_{* *}\right)} \\
\frac{2 \xi_{g \mu}}{\xi_{g g}} \sim\left[\frac{5 s-2}{b}\right] \frac{1+\bar{z}}{2}\left(\delta \chi H_{0}\right) \frac{\pi H_{0}}{k_{*}} \frac{\Delta^{2}\left(k_{*}\right)}{\Delta^{2}\left(k_{* *}\right)}
\end{gathered}
$$

The symbol $\Delta^{2}(k)$ denotes the dimensionless variance at scale $k$ and redshift $\bar{z}: 4 \pi k^{3} P_{m m}(k) /(2 \pi)^{3}$. Here, $k_{* *} \sim$ $1 / \sqrt{\delta \chi^{2}+\delta x_{\perp}^{2}}$, while $k_{*}$ is equal to either $1 / \delta x_{\perp}$ or $k_{m}$, whichever is smaller $\left(k_{m}\right.$ is the scale where $k^{2} P_{m m}(k)$ peaks; $k_{m} \gtrsim 3 \mathrm{~h} / \mathrm{Mpc}$ ). These estimates work reasonably well except for separations around or beyond the zerocrossing scale.

For the LOS orientation, where $\Delta^{2}\left(k_{*}\right)$ can be much larger than $\Delta^{2}\left(k_{* *}\right)$, the magnification bias corrections $\xi_{\mu \mu}$ and $\xi_{g \mu}$ can dominate over the intrinsic clustering correlation $\xi_{g g}$. The implications for baryon acoustic oscillation measurements are summarized at the end of \IB

\section{B. The Power Spectrum}

For surveys with a simple geometry, the power spectrum is often the more popular quantity to measure. Suppose the survey geometry is specified by $W(\mathbf{x})=$ $W_{\|}\left(x_{\|}\right) W_{\perp}\left(\mathbf{x}_{\perp}\right)$, where $\mathbf{x}$ specifies a location with $x_{\|}$being the LOS component and $\mathbf{x}_{\perp}$ the transverse component. For instance, a top-hat geometry in the radial direction is described by

$$
\begin{aligned}
W_{\|}\left(x_{\|}\right) & =1 / \sqrt{L} \text { if }-L / 2<x_{\|}<L / 2 \\
& =0 \text { otherwise }
\end{aligned}
$$

with $L$ being the radial span. A Gaussian geometry in the radial direction would be described by

$$
W_{\|}\left(x_{\|}\right)=\left(\pi \sigma^{2}\right)^{-1 / 4} \exp \left[-x_{\|}^{2} /\left(2 \sigma^{2}\right)\right]
$$

Our normalization convention is that $\int d^{3} x W^{2}=1$. Note that the origin $x_{\|}=0$ is chosen to be located at the mean redshift of interest.

The Fourier counterparts of eq. (7) and (8), taking into account the effects of the window, are

$$
2 P_{g \mu}(\mathbf{k})=\frac{3}{2} H_{0}^{2} \Omega_{m}(5 s-2)(1+\bar{z})
$$




$$
G\left(k_{\|}\right) \int \frac{d^{2} k_{\perp}^{\prime}}{(2 \pi)^{2}} P_{g m}\left(\bar{z}, k_{\perp}^{\prime}\right)\left|\tilde{W}_{\perp}\left(\mathbf{k}_{\perp}-\mathbf{k}_{\perp}^{\prime}\right)\right|^{2}
$$

and

$$
\begin{array}{r}
P_{\mu \mu}(\mathbf{k})=\left[\frac{3}{2} H_{0}^{2} \Omega_{m}(5 s-2)\right]^{2}\left|\tilde{W}_{\|}\left(k_{\|}\right)\right|^{2} \\
\int_{0}^{\bar{\chi}} d \chi^{\prime}\left(\bar{\chi}-\chi^{\prime}\right)^{2}\left(1+z^{\prime}\right)^{2} \\
\int \frac{d^{2} k_{\perp}^{\prime}}{(2 \pi)^{2}} P_{m m}\left(z^{\prime}, k_{\perp}^{\prime} \bar{\chi} / \chi^{\prime}\right)\left|\tilde{W}_{\perp}\left(\mathbf{k}_{\perp}-\mathbf{k}_{\perp}^{\prime}\right)\right|^{2}
\end{array}
$$

where $\tilde{W}_{\|}$and $\tilde{W}_{\perp}$ are Fourier transforms of the windows $W_{\|}$and $W_{\perp}$, and $G$ is defined as follows:

$$
\left.G\left(k_{\|}\right)=\int d x_{1} d x_{2}\left|x_{1}-x_{2}\right| e^{i k_{\|}\left(x_{1}-x_{2}\right)} W_{\|}\left(x_{1}\right) W_{\|}\left(x_{2}\right) 16\right)
$$

where $x_{1}$ and $x_{2}$ represent the LOS distance.

The galaxy power spectrum is windowed in the usual way:

$$
P_{g g}(\mathbf{k})=\int \frac{d^{3} k^{\prime}}{(2 \pi)^{3}} P_{g g}^{\text {true }}\left(k^{\prime}\right)\left|\tilde{W}\left(\mathbf{k}-\mathbf{k}^{\prime}\right)\right|^{2}
$$

where $\tilde{W}$ is the Fourier transform of the total window $W$, and $P_{g g}^{\text {true }}$ represents the true/unwindowed galaxy power spectrum.

The observed power spectrum is the sum:

$$
P_{\text {obs }}(\mathbf{k})=P_{g g}(\mathbf{k})+2 P_{g \mu}(\mathbf{k})+P_{\mu \mu}(\mathbf{k})
$$

where $P_{\text {obs }}(\mathbf{k})$ is defined to be

$$
\begin{array}{r}
P_{\mathrm{obs}}(\mathbf{k})=\int d^{3} x_{1} d^{3} x_{2}\left\langle\delta_{\mathrm{obs}}\left(\mathbf{x}_{\mathbf{1}}\right) \delta_{\mathrm{obs}}\left(\mathbf{x}_{\mathbf{2}}\right)\right\rangle \\
W\left(\mathbf{x}_{\mathbf{1}}\right) W\left(\mathbf{x}_{\mathbf{2}}\right) e^{i \mathbf{k} \cdot\left(\mathbf{x}_{\mathbf{1}}-\mathbf{x}_{\mathbf{2}}\right)}
\end{array}
$$

The survey window enters into these three contributions to the observed power in distinct ways. For the galaxy-galaxy power spectrum, the window function is convolved as usual under an integral with the true 3D power. For $P_{\mu \mu}$, only the transverse window function is convolved under an integral with the power spectrum $\left(P_{m m}\right)$. The LOS window function is not convolved under an integral at all: $P_{\mu \mu}$ is directly proportional to $\left|\tilde{W}_{\|}\right|^{2}$. For $P_{g \mu}$, it is also directly proportional to some generalized LOS window function $G\left(k_{\|}\right)$. This interesting behavior of the magnification-magnification and galaxymagnification power spectra can be traced to the unique anisotropies of their real space counterparts: referring back to eq. (10), the appearance of $\left|\tilde{W}_{\|}\right|^{2}$ in $P_{\mu \mu}$ is related to the fact that $g$ is independent of the LOS separation, and the appearance of $G$ in $P_{g \mu}$ is related to the linear dependence of $f \delta \chi$ on the LOS separation.

The precise form of the window functions depends on the exact geometry. For the example of the top-hat window (eq. 12]), they are

$$
\left|\tilde{W}_{\|}\left(k_{\|}\right)\right|^{2}=\frac{4}{L k_{\|}^{2}}\left[\sin \left(k_{\|} L / 2\right)\right]^{2}
$$

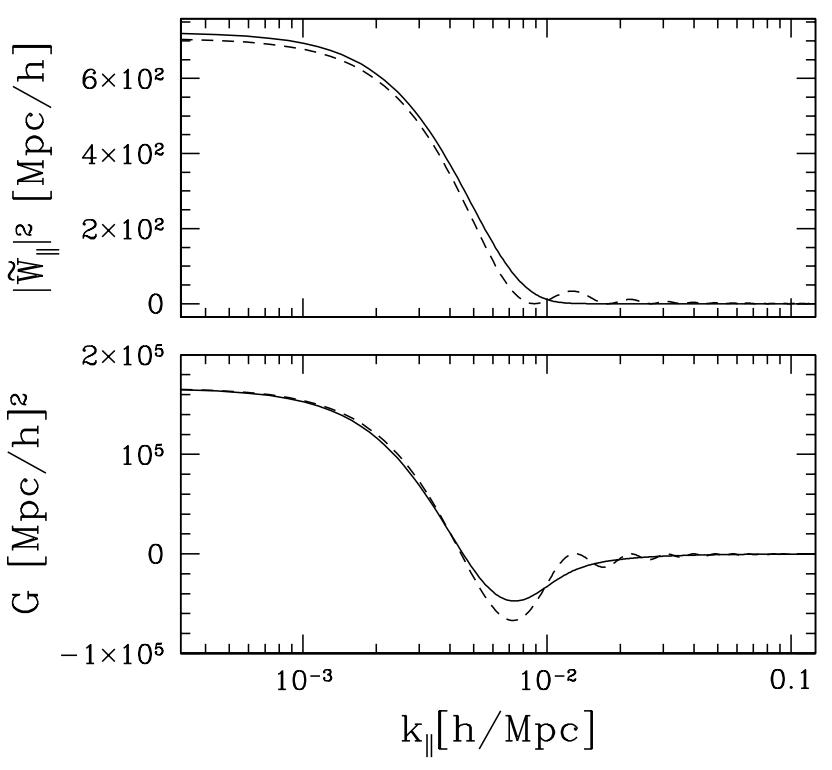

FIG. 1: The multiplicative LOS windows $\left|\tilde{W}_{\|}\right|^{2}$ and $G_{\|}$as a function of the LOS wavenumber $k_{\|}$. The dashed lines are for a top-hat geometry, with $L=706.6 \mathrm{Mpc} / \mathrm{h}$ and the solid lines are for a Gaussian geometry, with $\sigma=204 \mathrm{Mpc} / \mathrm{h}$.

$$
G\left(k_{\|}\right)=\frac{2}{k_{\|}^{2}}\left[\frac{2}{k_{\|} L} \sin \left(k_{\|} L\right)-\cos \left(k_{\|} L\right)-1\right]
$$

At low $k_{\|},\left|\tilde{W}_{\|}\right|^{2} \sim L$ while $G \sim L^{2} / 3$.

The corresponding expressions for the example of the Gaussian window (eq. 13]) are

$$
\begin{aligned}
& \left|\tilde{W}_{\|}\left(k_{\|}\right)\right|^{2}=\sqrt{4 \pi \sigma^{2}} e^{-\sigma^{2} k_{\|}^{2}} \\
& G\left(k_{\|}\right)=4 \sigma^{2}-8 k_{\|}^{2} \sigma^{4} \int_{0}^{1} d y e^{k_{\|}^{2} \sigma^{2}\left(y^{2}-1\right)}
\end{aligned}
$$

At low $k_{\|},\left|\tilde{W}_{\|}\right|^{2} \sim \sqrt{4 \pi} \sigma$, while $G \sim 4 \sigma^{2}$. Illustrations of $\left|\tilde{W}_{\|}\right|^{2}$ and $G$ can be found in Fig. 1 for both the top-hat and Gaussian geometries.

For completeness, let us also give the window functions in the transverse directions for a top-hat (a circle of radius $R$ ):

$$
\begin{aligned}
W_{\perp}\left(x_{\perp}\right) & =1 / \sqrt{\pi R^{2}} \text { if } x_{\perp}<R \\
& =0 \text { otherwise } \\
\tilde{W}_{\perp}\left(k_{\perp}\right) & =\left[\frac{4 \pi}{k_{\perp}^{4} R^{2}}\right]^{1 / 2} \int_{0}^{k_{\perp} R} d r r J_{0}(r) \\
& =\frac{\sqrt{4 \pi}}{k_{\perp}} J_{1}\left(k_{\perp} R\right)
\end{aligned}
$$

where $J_{0}$ and $J_{1}$ are the Bessel functions, and for a Gaussian:

$$
W_{\perp}\left(x_{\perp}\right)=\left(\pi \sigma^{2}\right)^{-1 / 2} \exp \left[-x_{\perp}^{2} /\left(2 \sigma^{2}\right)\right]
$$




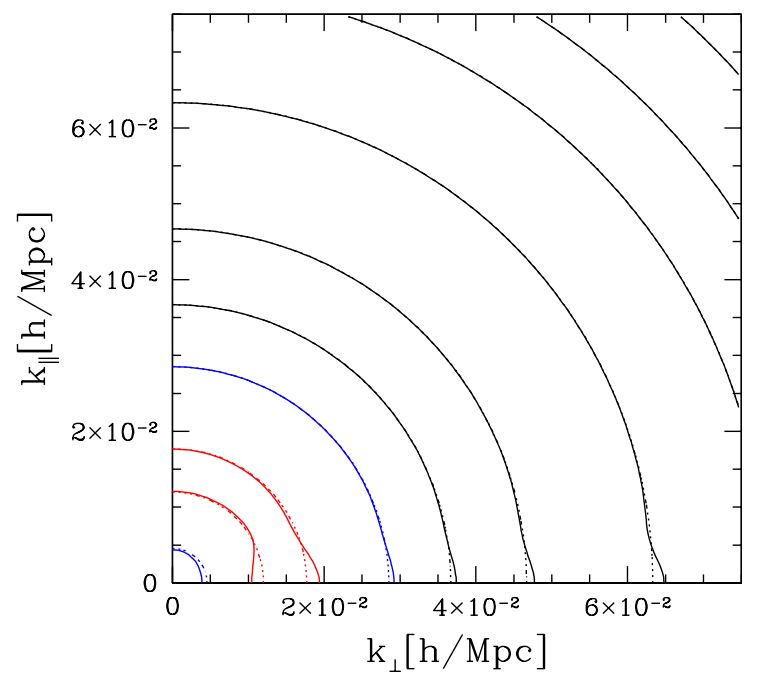

(a)

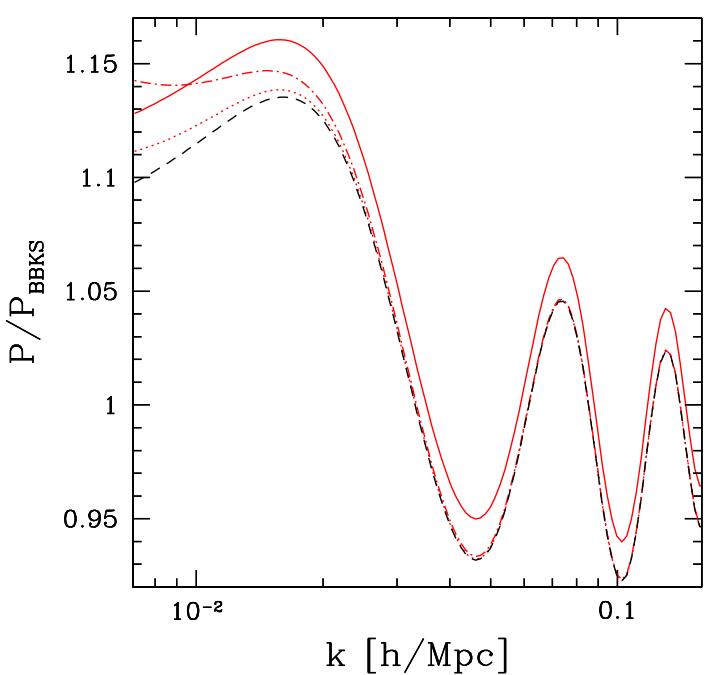

(b)

FIG. 2: $\bar{z}=1$ : (a) Contours of constant $P_{\text {obs }}\left(\right.$ solid) and $P_{g g}$ (dotted), left to right: $\log _{10}\left(P /[\mathrm{Mpc} / \mathrm{h}]^{3}\right)=4.02(\mathrm{red}$; double contours), 3.93 (blue: double contours), 3.83, 3.73, ... 3.33 (black). (b) Various power spectra normalized by the same BBKS (no baryon) galaxy power spectrum: $P_{\text {obs }}$ for $k_{\|}=0$ (red solid), $P_{g g}$ (black dashed), monopole of $P_{\text {obs }}((5 s-2) / b=1$ for red dotted and $(5 s-2) / b=2$ for red dot-dashed). Note that $k^{2}=k_{\|}^{2}+k_{\perp}^{2}$. A Gaussian window is assumed with $\sigma=204 \mathrm{Mpc} / \mathrm{h}$ for both panels, and $(5 s-2) / b=1$ is adopted throughout except for the red dot-dashed curve.

$$
\tilde{W}_{\perp}\left(k_{\perp}\right)=\sqrt{4 \pi \sigma^{2}} \exp \left[-\sigma^{2} k_{\perp}^{2} / 2\right]
$$

Note that in Fourier space the galaxy power spectrum $P_{g g}$ can itself be anisotropic if the window function is anisotropic. In the Gaussian case, if the same $\sigma$ were chosen for both $\tilde{W}_{\perp}$ and $\tilde{W}_{\|}$, the windowed galaxy power spectrum will remain isotropic. In our computations below in this section, we adopt this special choice in order to more clearly show the anisotropy induced by magnification bias. We have checked that using the top-hat geometry yields a rather similar lensing anisotropy as long as one makes the choice $2 R \sim L \sim \sqrt{12} \sigma$ (the latter equality is chosen such that the Gaussian $G$ and the top-hat $G$ have the same low $k_{\|}$limit).

As in the case of the correlation functions, it is useful to give order of magnitude estimates for the ratios $P_{\mu \mu} / P_{g g}$ and $P_{g \mu} / P_{g g}$ :

$$
\begin{aligned}
\frac{P_{\mu \mu}}{P_{g g}} & \sim\left[\frac{5 s-2}{b}\right]^{2} \frac{(1+\bar{z})^{2}}{50}\left(\bar{\chi} H_{0}\right)^{3} \frac{\left|\tilde{W}_{\|}\left(k_{\|}\right)\right|^{2}}{H_{0}^{-1}} \frac{P_{m m}\left(k_{\perp}\right)}{P_{m m}(k)} \\
\frac{2 P_{g \mu}}{P_{g g}} & \sim\left[\frac{5 s-2}{b}\right] \frac{1+\bar{z}}{2} H_{0}^{2} G\left(k_{\|}\right) \frac{P_{m m}\left(k_{\perp}\right)}{P_{m m}(k)}
\end{aligned}
$$

where $k^{2}=k_{\perp}^{2}+k_{\|}^{2}$. The above expressions are approximate. For instance, we have approximated $P_{m m}\left(k_{\perp} \bar{\chi} / \chi^{\prime}\right)$ (eq. 15) by $P_{m m}\left(k_{\perp}\right)$. Nonetheless, they agree with the exact numerical integration to within factor of a few, and they illustrate several important points.

The presence of $\left|\tilde{W}_{\|}\right|^{2}$ and $G$ means that the effects of magnification bias are largest for low $k_{\|}$'s. The simplest limit to consider is the one with $k_{\perp} \gg k_{\|}$(i.e. a $\mathbf{k}$ vector that is oriented transverse to the LOS) in which case the factors of $P_{m m}$ cancel out in the ratios, since $k \sim k_{\perp}$. In the small $k_{\|}$limit, $\left|\tilde{W}_{\|}\right|^{2} \sim L$ and $G \sim L^{2} / 3$, where $L$ is the width of the redshift bin over which one is measuring the power spectrum. One can see that for instance at $\bar{z} \sim 1.5$ where $\bar{\chi} H_{0} \sim 1$, and for $(5 s-2) / b \sim 1$, we have $P_{\mu \mu} / P_{g g} \sim 0.1 H_{0} L$ and $2 P_{g \mu} / P_{g g} \sim 0.4\left(H_{0} L\right)^{2}$. A choice of $L \sim 900 \mathrm{Mpc} / \mathrm{h}$ (which corresponds to the redshift interval $1.5 \pm 0.35)$ yields $P_{\mu \mu} / P_{g g} \sim 0.03$ and $2 P_{g \mu} / P_{g g} \sim 0.04$. In other words, the total effect of magnification bias is quite modest, $\sim 7 \%$ in this configuration. It should be kept in mind that (1) this estimate increases with $L$, and (2) the $P_{\mu \mu} / P_{g g}$ ratio increases strongly with redshift: the cubic dependence on $\left(\bar{\chi} H_{0}\right)$ and quadratic dependence on $1+\bar{z}$ means this ratio rises rapidly beyond $\bar{z} \sim 1.5$.

Nonetheless, it is perhaps a little surprising that magnification bias appears to have a much more modest effect on the observed clustering in Fourier space compared to real space, where the corresponding ratios $\xi_{\mu \mu} / \xi_{g g}$ and $2 \xi_{g \mu} / \xi_{g g}$ can reach order unity or even higher in the LOS orientation (eq. 11; see paper I for details). The fundamental reason is the absence in Fourier space of this potentially large boost factor, $\Delta^{2}\left(k_{*}\right) / \Delta^{2}\left(k_{* *}\right)$ in eq. (11), that is present for the correlation function. Consider a separation vector $\delta \mathbf{x}$ that is oriented along the LOS, which is the orientation that maximizes the magnification 


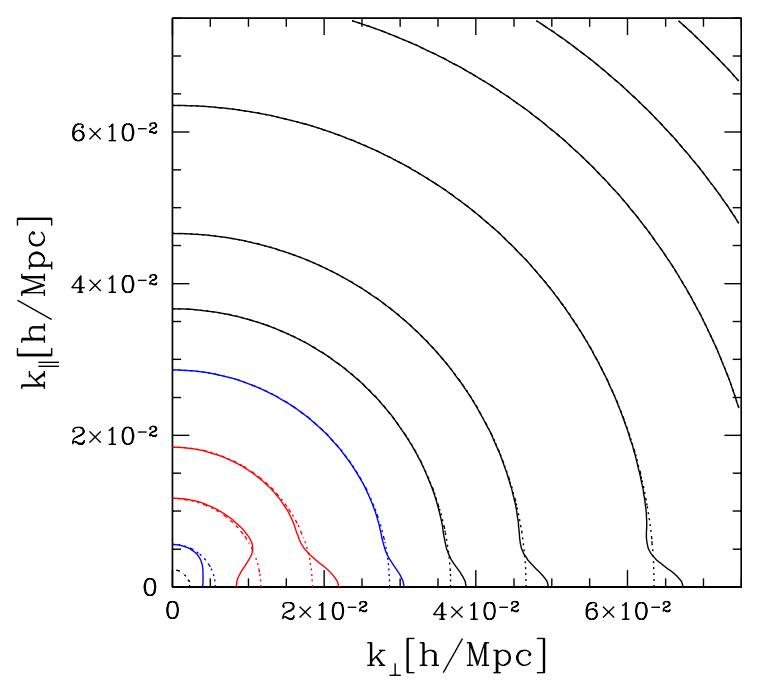

(a)

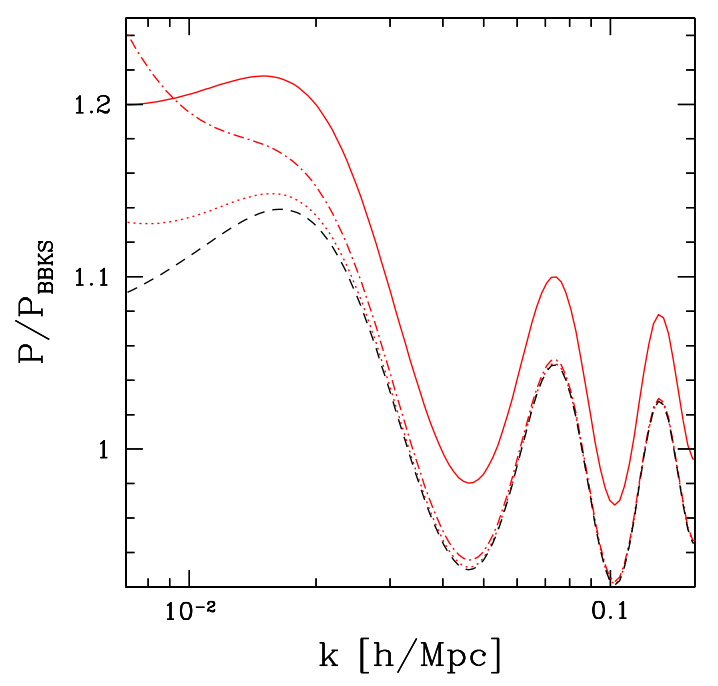

(b)

FIG. 3: Analog of Fig. 2 for $\bar{z}=1.5$. The contours in (a) are, left to right: $\log _{10}\left(P /[\mathrm{Mpc} / \mathrm{h}]^{3}\right)=3.86$ (red; double contours), 3.76 (blue: double contours), 3.66, 3.56, ... 3.16 (black). A Gaussian window is assumed with $\sigma=270 \mathrm{Mpc} / \mathrm{h}$.

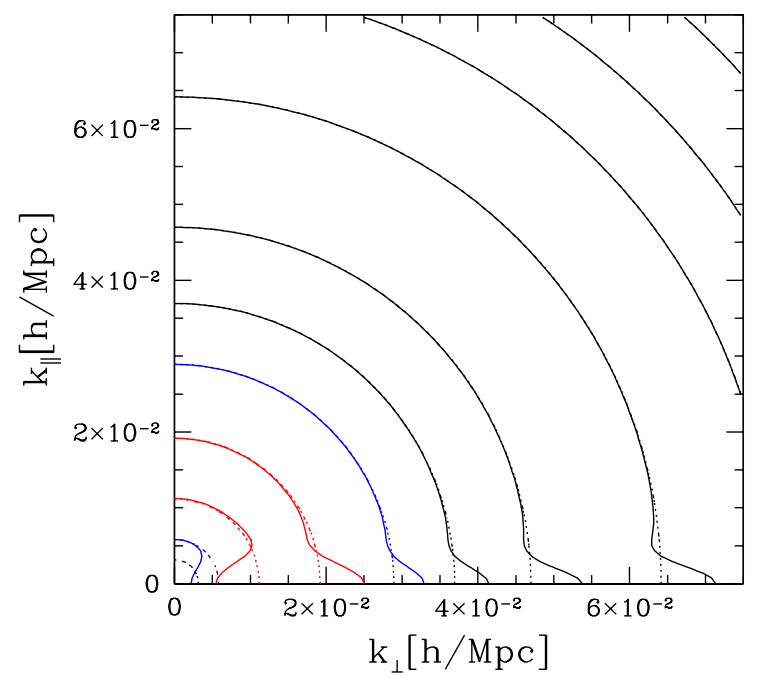

(a)

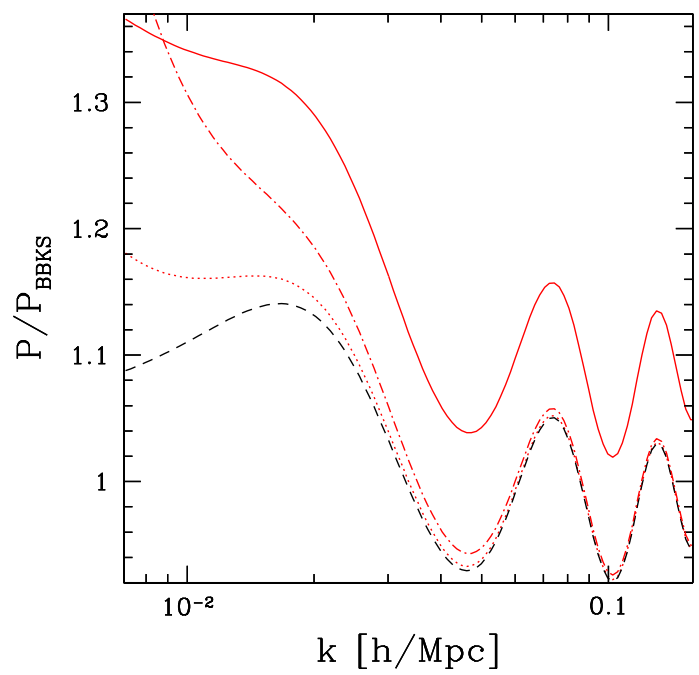

(b)

FIG. 4: Analog of Fig. 2 for $\bar{z}=2$. The contours in (a) are, left to right: $\log _{10}\left(P /[\mathrm{Mpc} / \mathrm{h}]^{3}\right)=3.71$ (red; double contours), 3.61 (blue: double contours), 3.51, 3.41, .. 3.01 (black). A Gaussian window is assumed with $\sigma=323 \mathrm{Mpc} / \mathrm{h}$.

bias effect. If $|\delta \mathbf{x}|$ is sufficiently large, the galaxy-galaxy correlation $\xi_{g g}$ is essentially determined by the power spectrum in the linear regime (low $k_{* *} \sim 1 /|\delta \mathbf{x}|$ in eq. [11) and is quite weak, while the galaxy-magnification correlation $\xi_{g \mu}$ and the magnification-magnification correlation $\xi_{\mu \mu}$ are sensitive to the power in the nonlinear regime (high $k_{*} \sim 1 / \delta x_{\perp}$ in eq. 111) and can be appre- ciable. In other words, in the LOS orientation in real space, one is comparing intrinsic galaxy fluctuations and lensing fluctuations on very different scales.

Consider, on the other hand, a vector $\mathbf{k}$ that points in the transverse direction, which is the Fourier analog of a LOS $\delta \mathbf{x}$. In this case, $k \sim k_{\perp}$ and factors of the mass power spectrum simply cancel out of the ratios in 


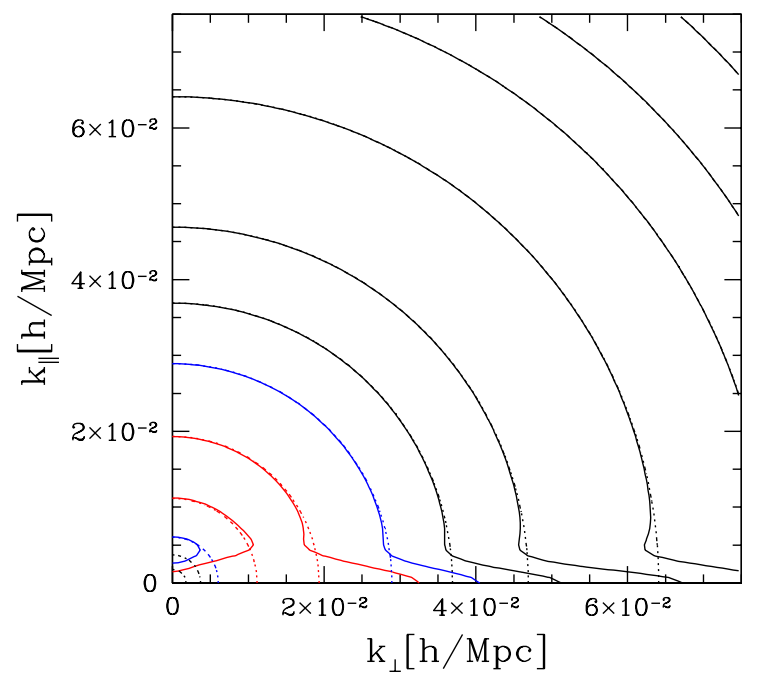

(a)

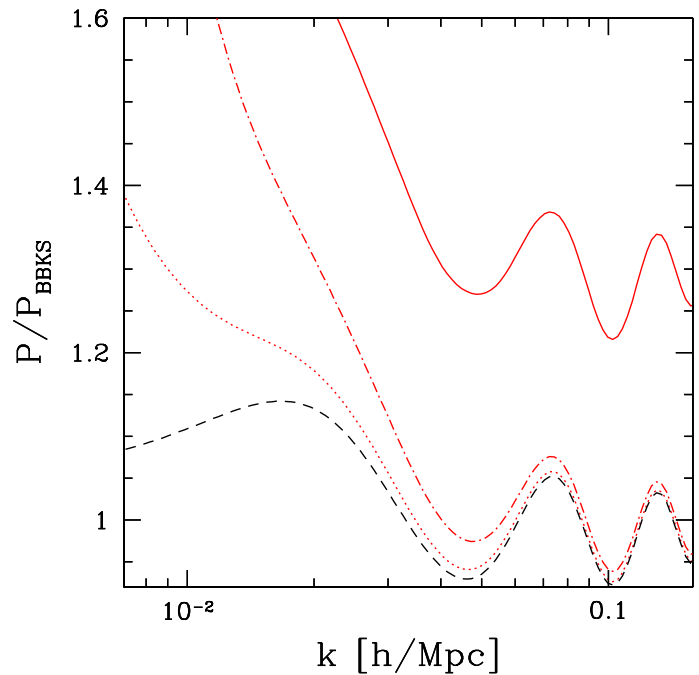

(b)

FIG. 5: Analog of Fig. 2 for $\bar{z}=3$. The contours in (a) are, left to right: $\log _{10}\left(P /[\mathrm{Mpc} / \mathrm{h}]^{3}\right)=3.47$ (red; double contours), 3.37 (blue: double contours), 3.27, 3.17, ... 2.77 (black). A Gaussian window is assumed with $\sigma=397 \mathrm{Mpc} / \mathrm{h}$.

eq. (24). There is no boost coming from a ratio of powers on very different scales, as in the case of the correlation function. In other words, the mixing of magnification bias corrections with the intrinsic galaxy clustering term occurs in a very different manner in Fourier space than in real space.

Ultimately, the correlation function and the power spectrum are related by Fourier transform, and so should really contain the same information. The important point to keep in mind, however, is that for cosmological purposes, one often focuses on scales that are perceived to be linear: in the case of the correlation function, that means a large $|\delta \mathbf{x}|$, and in the case of the power spectrum, that means a small $|\mathbf{k}|$. With the presence of magnification bias, a large $|\delta \mathbf{x}|$ no longer protects one against nonlinearity i.e. a separation $\delta \mathbf{x}$ pointing along the LOS (i.e. small $\left|\delta \mathbf{x}_{\perp}\right|$ ) is subject to large magnification corrections even if $|\delta \mathbf{x}|$ is large. On the other hand, a small $|\mathbf{k}|$ means both $k_{\|}$and $\left|\mathbf{k}_{\perp}\right|$ must be small, which protects one against nonlinear fluctuations.

Let us return to the order of magnitude estimates in eq. (24), to see what happens if one considers angular averages. The monopole of any power spectrum $P$ is defined as

$$
\text { monopole of } P(\mathbf{k})=\int_{0}^{\pi / 2} P(\mathbf{k}) \sin \theta_{k} d \theta_{k} .
$$

where $\theta_{k}$ is the angle between the LOS and $\mathbf{k}$. Let us focus on the monopole $P_{\mu \mu} / P_{g g}$ for a Gaussian window, where the calculation is the simplest. Suppose one is interested in a scale $k$ such that $k \sigma \gtrsim 3$. Because of the Gaussian in $\left|\tilde{W}_{\|}\right|^{2}$, the integral over angle will be dominated by $\theta_{k} \sim \pi / 2$, and so one can approximate $P_{m m}\left(k_{\perp}\right)$ by $P_{m m}(k)$. The remaining integral over angle is simple to do, and yields the ratio:

$$
\frac{\text { monopole of } P_{\mu \mu}}{\text { monopole of } P_{g g}} \sim\left[\frac{5 s-2}{b}\right]^{2} \frac{(1+\bar{z})^{2}}{50}\left(\bar{\chi} H_{0}\right)^{3} \frac{\pi H_{0}}{k}(26)
$$

which is valid only for $k \sigma \gtrsim 3$. Note how the width of the window $\sigma$ completely disappears from this ratio. A similar expression holds for the tophat case as well. This ratio is small for $k / H_{0} \gg 1$, unless one goes to a sufficiently high redshift. The corresponding ratio for galaxymagnification cannot be worked out analytically because the window $G$ has a more complicated form. On dimensional grounds, one expects this ratio to scale with $\sigma$. In practice, we find that for redshifts where magnification bias matters, the monopole of the galaxy-magnification power spectrum is quite a bit smaller than that of the magnification-magnification power spectrum, in part because of cancellations that occur under the angular average.

The intuition gained above from the order of magnitude estimates (eq. 24, 26]) is confirmed by the exact numerical evaluation of $P_{g \mu}, P_{\mu \mu}$ and $P_{g g}$ according to eq. (14), (15) and (17). This is shown in Fig. 2 - 5 for redshifts $\bar{z}=1,1.5,2,3$. The magnification distortion increases with redshift and, as discussed before, has the most noticeable effects for small $k_{\|}$'s. Exactly how small $k_{\|}$needs to be to see a substantial effect depends on the LOS width of the survey/sample selection function. For a Gaussian window function the region of large magnification distortion is $k_{\|} \lesssim \sigma^{-1}$. Panels (b) of Fig. 2] - 5 show that the monopole of the power spectrum is visi- 
bly distorted as well, the effect at low $k$ 's is particularly severe at redshifts $\gtrsim 2$.

There is the interesting question of how magnification bias impacts baryon oscillation measurements. This was addressed in paper I for the real space correlation function. Briefly summarizing: we found that the observed baryon acoustic scale can be shifted by up to $\sim 3 \%$ in the LOS orientation, and up to $\sim 0.6 \%$ in the monopole, depending on the exact values of the galaxy bias, redshift and number count slope. The corresponding shifts in the inferred Hubble parameter and angular diameter distance, if ignored, could significantly bias measurements of the dark energy equation of state (by up to $\sim 15 \%$ ). In Fourier space, there are several wiggles, and the magnification bias induced shift in the baryon oscillation scale is likely more sensitive to exactly how this scale is extracted from data. We therefore do not attempt to investigate this further in this paper. Given the earlier discussions, it is reasonable to expect that baryon acoustic oscillation measurements are less affected by magnification bias in Fourier space. However, it is worth emphasizing that magnification bias introduces scale and orientation dependent corrections to the observed power spectrum, and these corrections depend on uncertain factors such as the galaxy bias. The question is whether, in fitting the observed data for the baryon oscillation scale, one should introduce additional fit parameters to account for magnification bias, and what impact they might have on the measurement accuracy of the Hubble parameter and the angular diameter distance. This certainly deserves more study.

It is also worth noting that, as can be seen from panels (b) of Fig. 22-5, the radiation-matter equality peak location/shape around $k \sim 0.01 \mathrm{~h} / \mathrm{Mpc}$ is likely significantly affected by magnification bias, and one must be careful in using it as a standard ruler [28].

\section{INCORPORATING REDSHIFT DISTORTION DUE TO PECULIAR MOTIONS}

The observed redshift of a source galaxy is dependent upon both the distance to the source (the cosmological redshift) and the peculiar velocity of the source. In redshift space, the observed galaxy density is (to first order in perturbations):

$$
\delta_{\mathrm{obs}}=\delta_{g}+\delta_{\mu}+\delta_{v}
$$

where $\delta_{g}$ and $\delta_{\mu}$ are as in eq. (1), and $\delta_{v}$ is

$$
\delta_{v}=-\frac{(1+\bar{z})}{H(\bar{z})} \frac{\partial v_{\|}}{\partial x_{\|}}
$$

where $v_{\|}$is the LOS peculiar velocity, and $H(\bar{z})$ is the Hubble parameter at the mean redshift $\bar{z}$.

The observed two-point correlation function, instead of eq. (6), is now given by

$$
\xi_{\text {obs }}(1 ; 2)=\xi_{g g}(1 ; 2)+\xi_{g \mu}(1 ; 2)+\xi_{g \mu}(2 ; 1)
$$

$$
+\xi_{\mu \mu}(1 ; 2)+\xi_{g v}(1 ; 2)+\xi_{v g}(2 ; 1)+\xi_{v v}(1 ; 2)
$$

where we have used the arguments 1 and 2 as the shorthand for the positions of the two points of interest in redshift-space. We have used the Limber approximation which makes the velocity-magnification cross-terms vanish (the derivative with respect to $x_{\|}$in the velocity term pulls down a factor of $k_{\|}$, which vanishes under the Limber approximation; see [2]). The galaxy-velocity crosscorrelation and the velocity auto-correlation are given by the well-known results of Kaiser [29]:

$$
\xi_{g v}(1,2)=\left[\frac{a}{a^{\prime}} \frac{D^{\prime}}{D}\right] \int \frac{d^{3} k}{(2 \pi)^{3}} \frac{k_{\|}^{2}}{k^{2}} P_{g m}(k) e^{i \mathbf{k} \cdot\left(\mathbf{x}_{1}-\mathbf{x}_{\mathbf{2}}\right)}
$$

and

$$
\xi_{v v}(1,2)=\left[\frac{a}{a^{\prime}} \frac{D^{\prime}}{D}\right]^{2} \int \frac{d^{3} k}{(2 \pi)^{3}} \frac{k_{\|}^{4}}{k^{4}} P_{m m}(k) e^{i \mathbf{k} \cdot\left(\mathbf{x}_{1}-\mathbf{x}_{2}\right)}
$$

where $\mathbf{x}_{\mathbf{1}}-\mathbf{x}_{\mathbf{2}}$ has a LOS component $\chi_{1}-\chi_{2}$, and transverse components of $\bar{\chi}\left(\boldsymbol{\theta}_{1}-\boldsymbol{\theta}_{2}\right)$. Here, $a$ is the scale factor, $D$ is the linear growth factor, and $a^{\prime}$ and $D^{\prime}$ are their derivatives with respect to conformal time. All time dependent quantities are evaluated at the mean redshift $\bar{z}$.

The observed power spectrum, instead of eq. (18), is now given by

$$
\begin{aligned}
& P_{\text {obs }}(\mathbf{k})=P_{g g}(\mathbf{k})+2 P_{g \mu}(\mathbf{k})+P_{\mu \mu}(\mathbf{k}) \\
& \quad+2 P_{g v}(\mathbf{k})+P_{v v}(\mathbf{k})
\end{aligned}
$$

where the first line is as before (eq. [14, 15] \& [17]), and

$$
P_{g v}(\mathbf{k})=\left[\frac{a}{a^{\prime}} \frac{D^{\prime}}{D}\right] \int \frac{d^{3} k^{\prime}}{(2 \pi)^{3}} \frac{k_{\|}^{\prime 2}}{{k^{\prime}}^{2}} P_{g m}\left(k^{\prime}\right)\left|\tilde{W}\left(\mathbf{k}-\mathbf{k}^{\prime}\right)\right|^{2}(33)
$$

and

$$
\left.P_{v v}(\mathbf{k})=\left[\frac{a}{a^{\prime}} \frac{D^{\prime}}{D}\right]^{2} \int \frac{d^{3} k^{\prime}}{(2 \pi)^{3}} \frac{k_{\|}^{\prime 4}}{{k^{\prime}}^{4}} P_{m m}\left(k^{\prime}\right)\left|\tilde{W}\left(\mathbf{k}-\mathbf{k}^{\prime}\right)\right|^{2} 34\right)
$$

To gain some intuition about the various effects at work, it is useful to adopt the following approximation: integrate out all the convolving windows as if they are delta functions. (This approximation is made only in this section, not in previous sections.) For instance:

$$
\int \frac{d^{3} k^{\prime}}{(2 \pi)^{3}} P\left(k^{\prime}\right)\left|\tilde{W}\left(\mathbf{k}-\mathbf{k}^{\prime}\right)\right|^{2} \sim P(k)
$$

where $P$ represents $P_{g g}, P_{g m}, P_{g m} k_{\|}^{\prime 2} / k^{\prime 2}$ and so on, and the convolving window $\tilde{W}$ could also be $\tilde{W}_{\perp}$. This works well if the power spectrum is sufficiently smooth and the convolving window is sufficiently narrow. With this approximation, we obtain

$$
P_{\text {obs }}(\bar{z}, \mathbf{k})=P_{g g}(\bar{z}, k)\left[\left(1+\frac{f_{D}}{b} \frac{k_{\|}^{2}}{k^{2}}\right)^{2} e^{-k_{\|}^{2} \sigma_{z}^{2}}+\right.
$$




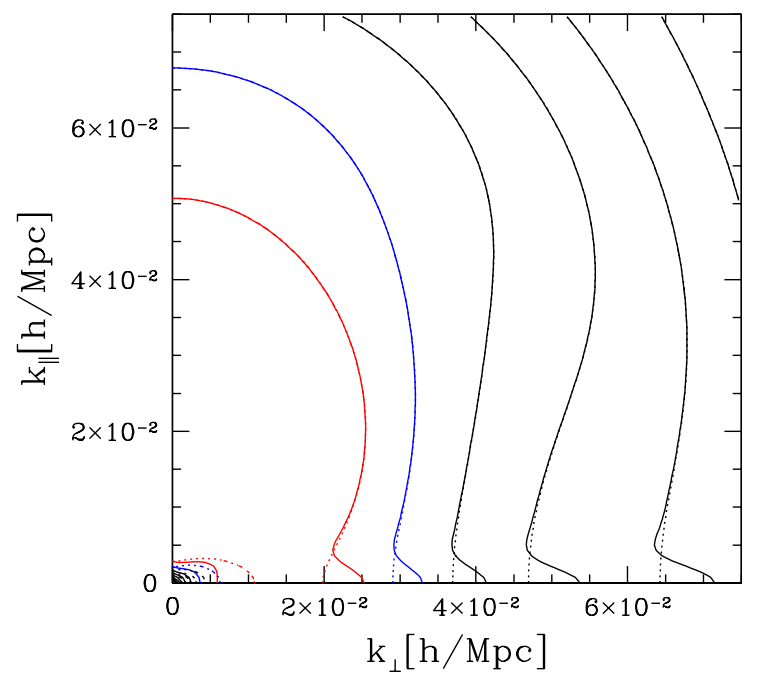

(a)

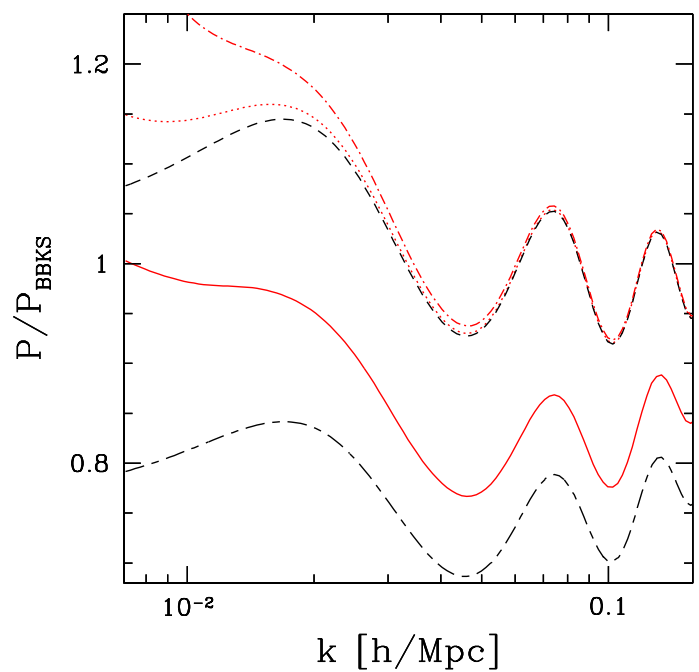

(b)

FIG. 6: Analog of Fig. 4 for $\bar{z}=2$, except redshift distortion is incorporated with $f_{D} / b=0.475$, and $\sigma_{z}=3 \mathrm{Mpc} / \mathrm{h}$ (eq. [36). (a) Contours of constant $P_{\text {obs }}$ with magnification bias (solid) and without magnification bias (dotted), left to right: $\log _{10}\left(P /[\mathrm{Mpc} / \mathrm{h}]^{3}\right)=3.71$ (red; double contours), 3.61 (blue: double contours), 3.51, 3.41, 3.31, 3.21 (black). (b) Various power spectra normalized by the same BBKS (no baryon) galaxy monopole power spectrum: $P_{\text {obs }}$ with magnification bias for $k_{\|}=0$ (red solid), $P_{\text {obs }}$ without magnification bias for $k_{\|}=0$ (black short-long dashed), monopole of $P_{\text {obs }}$ without magnification bias (black uniform dashed), monopole of $P_{\text {obs }}$ with magnification bias $((5 s-2) / b=1$ for red dotted and $(5 s-2) / b=2$ for red dot-dashed). Note that $k^{2}=k_{\|}^{2}+k_{\perp}^{2}$. A Gaussian window is assumed with $\sigma=323 \mathrm{Mpc} / \mathrm{h}$ for both panels, and $(5 s-2) / b=1$ is adopted throughout except for the red dot-dashed curve.

$$
\begin{aligned}
& q(1+\bar{z}) G\left(k_{\|}\right) \frac{P_{m m}\left(\bar{z}, k_{\perp}\right)}{P_{m m}(\bar{z}, k)}+ \\
& \left.q^{2}\left|\tilde{W}_{\|}\left(k_{\|}\right)\right|^{2} \int_{0}^{\bar{\chi}} d \chi^{\prime}\left(\bar{\chi}-\chi^{\prime}\right)^{2}\left(1+z^{\prime}\right)^{2} \frac{P_{m m}\left(z^{\prime}, k_{\perp} \bar{\chi} / \chi^{\prime}\right)}{P_{m m}(\bar{z}, k)}\right]
\end{aligned}
$$

where

$$
f_{D} \equiv \frac{d \ln D}{d \ln a}
$$

evaluated at the mean redshift $\bar{z}$, and

$$
q \equiv \frac{3}{2} H_{0}^{2} \Omega_{m} \frac{(5 s-2)}{b}
$$

and we have assumed a linear galaxy bias $b$ (at redshift $\bar{z}$ ). We have introduced an exponential factor $\exp \left[-k_{\|}^{2} \sigma_{z}^{2}\right]$ which accounts for a possible (Gaussian) dispersion in redshifts, such as from photometric redshifts. Here $\sigma_{z}$ is the dispersion expressed in comoving $\mathrm{Mpc} / \mathrm{h}$ (not in redshift). One can also think of this exponential factor as modeling the effect of nonlinear or virialized peculiar motions, though this description is at best approximate [30]. Strictly speaking, with a non-zero $\sigma_{z}$, the multiplicative window $G$ in eq. (36) should be replaced by

$$
\begin{aligned}
& G_{z}\left(k_{\|}\right)=\int \frac{d z_{1} d z_{2} d z_{1}^{\prime} d z_{2}^{\prime}}{2 \pi \sigma_{z}^{2}}\left|z_{1}^{\prime}-z_{2}^{\prime}\right| e^{i k_{\|}\left(z_{1}-z_{2}\right)} \\
& W_{\|}\left(z_{1}\right) W_{\|}\left(z_{2}\right) \exp \left[-\frac{\left(z_{1}^{\prime}-z_{1}\right)^{2}}{2 \sigma_{z}^{2}}\right] \exp \left[-\frac{\left(z_{2}^{\prime}-z_{2}\right)^{2}}{2 \sigma_{z}^{2}}\right]
\end{aligned}
$$

where $z_{1}, z_{2}, z_{1}^{\prime}, z_{2}^{\prime}$ denotes LOS distances, not redshifts. We find that as long as the LOS width of the survey/sample selection $W_{\|}$is large compared to $\sigma_{z}$ (i.e. $L \gg \sigma_{z}$ for a top-hat geometry, or $\sigma \gg \sigma_{z}$ for a Gaussian geometry; see eq. 20] \& 21]), the above $G_{z}$ is well approximated by $G$ as defined in eq. (16).

The first line of eq. (36), excluding the exponential factor, represents the classic Kaiser distortion due to coherent infall. The second and third lines come from the galaxy-magnification and magnificationmagnification correlations respectively. We illustrate all these effects in Fig. 6 and 7 by evaluating eq. (36) for $\bar{z}=2$. Fig. 6 uses $\sigma_{z}=3 \mathrm{Mpc} / \mathrm{h}$, which corresponds to a velocity dispersion of $\sim 300 \mathrm{~km} / \mathrm{s}$, or a redshift dispersion of 0.003. This is the level of dispersion one expects from virialized motions on small scales.

For the scales shown in Fig. 6, the Kaiser distortion dominates over the finger-of-god effect due to virialized motions: the contours of constant $P_{\text {obs }}$ are elongated in the LOS direction for $k$ 's larger than the radiationmatter equality scale, and compressed otherwise. It is also worth noting that magnification distortion survives the Kaiser distortion. The two kinds of distortions have fundamentally different shapes. Magnification distortion is localized to small $k_{\|}$'s, whereas the Kaiser distortion is more spread out. The localized nature of magnification distortion originates from the fact that the corrections 


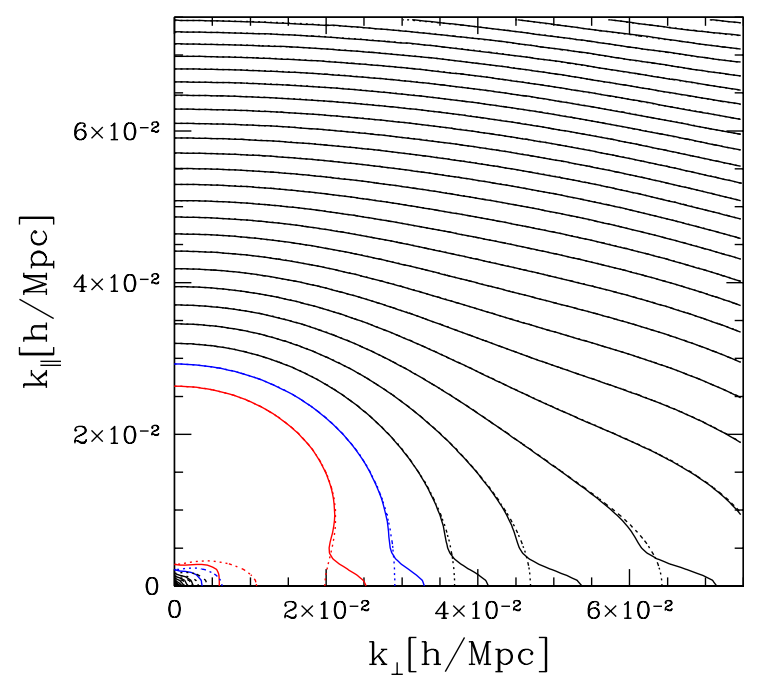

(a)

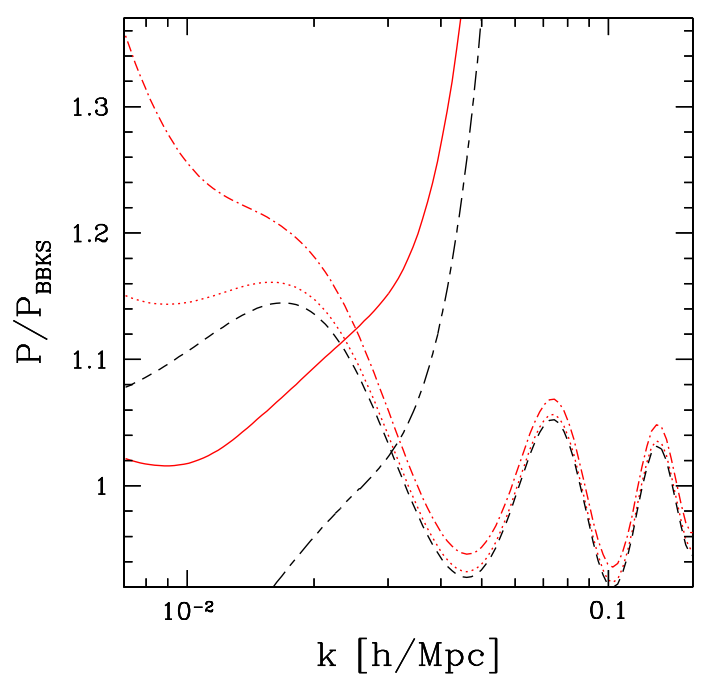

(b)

FIG. 7: Analog of Fig. 6 except that $\sigma_{z}=30 \mathrm{Mpc} / \mathrm{h}$. The contours in (a) are, left to right: $\log _{10}\left(P /[\mathrm{Mpc} / \mathrm{h}]^{3}\right)=3.71(\mathrm{red}$; double contours), 3.61 (blue: double contours), 3.51, 3.41, .. 1.01 (black). In panel (b), note how the monopole (red dotted with magnification bias and black uniform dashed without) is higher than the $k_{\|}=0$ power (red solid with magnification bias and black short-long dashed without) for small $k$ 's due to the Kaiser effect, and lower for high $k$ 's due to the finger-of-god effect (from the large $\sigma_{z}$ ).

it introduces (the second and third terms on the right hand side of eq. 36]) are proportional to the multiplicative windows $G\left(k_{\|}\right)$and $\left|\tilde{W}_{\|}\left(k_{\|}\right)\right|^{2}$, both of which peak at small $k_{\|}$'s.

Interestingly, the Kaiser distortion vanishes at $k_{\|}=0$, exactly where magnification bias has the largest effect. This suggests it should be possible to disentangle the two different distortions from data. For instance, the one free parameter that controls the Kaiser distortion, $f_{D} / b$, can be determined from the observed power spectrum anisotropy by excluding from consideration the small $k_{\|}$ modes. With this in hand, one should be able to predict the dotted contours such as those in Fig. 6a. The difference between the observed (solid) contours and the dotted ones then gives us the magnification bias corrections. The galaxy-magnification and magnificationmagnification contributions can be further separated from each other by using the distinctive shapes of their respective multiplicative windows $G$ and $\left|\tilde{W}_{\|}\left(k_{\|}\right)\right|^{2}$ (for instance, the latter is positive definite whereas the former can go negative; see Fig. 1).

Fig. 7 is analogous to Fig. 6 except that $\sigma_{z}$ is increased to $30 \mathrm{Mpc} / \mathrm{h}$, corresponding to a velocity dispersion of $\sim 3000 \mathrm{~km} / \mathrm{s}$, or a redshift dispersion of 0.03 . This larger value for $\sigma_{z}$ is chosen to mimic the effect of photometric redshifts. In contrast to the case of $\sigma_{z}=3$ $\mathrm{Mpc} / \mathrm{h}$, one can clearly see here a finger-of-god effect of sorts: the contours of constant $P_{\text {obs }}$ are compressed in the LOS direction, for sufficiently large $k$ 's. As before, magnification distortion is clearly visible, being well localized to small $k_{\|}$'s. Its distinctive shape makes it in principle distinguishable from both the Kaiser distortion and the finger-of-god effect, which are more spread out on the $k_{\perp}-k_{\|}$plane.

It is also worth noting that in cases where the power spectrum is strongly anisotropic, such as in Fig. 7, the monopole is probably not the most relevant quantity to consider. This is because some orientations are much more noisy than the others, and one might not want to weigh them equally (or more precisely, according to eq. 25]). In general, with sufficient redshift accuracy, one should make use of the full 3D information available. In this vein, it is not uncommon to consider higher multipoles of the anisotropic power spectrum [31]. This is especially useful for analyzing the Kaiser distortion since it gives rise to only 2 extra multipoles. However, a multipole expansion is likely not helpful in analyzing magnification distortion, due to its localized nature. It is probably more useful to take advantage of the special dependence on $G\left(k_{\|}\right)$and $\left|\tilde{W}_{\|}\left(k_{\|}\right)\right|^{2}$ of the galaxymagnification and magnification-magnification terms (eq. 36). How to optimally extract these two contributions from noisy data deserves further study.

In addition to redshift distortion due to peculiar motions, another well known effect is the so called cosmological distortion, or Alcock-Paczynski effect [32, 33]. This is taken up in Appendix A. 


\section{DISCUSSION}

In paper I, we examined the effects of magnification distortion in real/configuration space, and here we have extended the analysis to Fourier and redshift space. The observed galaxy/quasar correlation is endowed with a distinctive lensing induced anisotropy. This is encapsulated in eq. (10) for real space and eq. (14), (15), (17) and (18) for Fourier space: the linear dependence on the LOS separation $\delta \chi$ in the real space galaxymagnification correlation gives rise to the multiplicative window $G$ in the galaxy-magnification power spectrum. Likewise, the independence of the real space magnification-magnification correlation on $\delta \chi$ accounts for the appearance of the multiplicative window $\left|\tilde{W}_{\|}\right|^{2}$ in the magnification-magnification power spectrum.

Qualitatively, the galaxy correlation becomes enhanced for the transverse modes in Fourier space, and for pairs oriented along the LOS in real space. Quantitatively, the degree of enhancement is rather different for

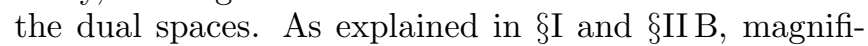
cation distortion is less severe in Fourier space: as long as one focuses on modes with a small wavenumber $k$, as is usually done when obtaining cosmological constraints, both the intrinsic galaxy fluctuations and the lensing fluctuations are in the linear regime. In real space, even if the pair separation is large, one cannot help but mix up linear intrinsic galaxy fluctuations with nonlinear lensing fluctuations, as long as one considers the LOS orientation. Incidentally, this implies that a linear galaxy bias is a better approximation in Fourier space than in real space.

The above findings suggest that in precision measurements of the galaxy power spectrum, such as those that attempt to use the baryon oscillation scale to constrain dark energy [14, 15, 16, 17, 18, 19, 20], the simplest way to immunize against magnification bias could be to go to Fourier space, and remove from consideration the small $k_{\|}$(transverse) modes where magnification bias has the largest effect. However, in a photometric redshift survey, these are probably the modes with the highest signalto-noise, and the Fourier space also suffers from possible complications due to a non-Poissonian shot noise [34]. A better strategy is perhaps to face the magnification bias corrections head on, and use the full 3D information to constrain and measure them - after all, they contain interesting cosmological information too.

Precisely how magnification bias corrections might shift the baryon oscillation scale is a subject worthy of a separate paper. The precise shift will depend on exactly how the oscillation scale is extracted from data. A preliminary investigation in real space, where the acoustic oscillations manifest as a single local maximum whose position is easily defined, was presented in paper I: as discussed at the end of $₫ \mathrm{IIB}$, the impact on measurements of the dark energy equation of state can be significant (shifting it by up to $\sim 15 \%$ ). It should also be emphasized that the baryon oscillations are not the only large scale features of interest. The radiation-matter equality peak at around $k \sim 0.01 \mathrm{~h} / \mathrm{Mpc}$ contains valuable cosmological information, but it can be seen from Fig. 20-5 panels (b) that the power spectrum at this scale is likely significantly affected by magnification bias.

We have incorporated the effects of peculiar motion (and redshift inaccuracy) on the power spectrum anisotropy in $\$$ (the Alcock-Paczynski effect is further incorporated in Appendix $\mathrm{A}$ ). The main conclusion is that the lensing induced features remain rather robust, thanks to their localized nature to small $k_{\|}$'s. It should be in principle possible to separately measure from data the galaxy-galaxy, galaxy-magnification and magnificationmagnification power spectra, exploiting their different shapes in the $k_{\|}-k_{\perp}$ plane (see eq. 36). Exactly how to do so in an optimal fashion when faced with noisy data deserves further study. In particular, different galaxy types have a different galaxy bias and number count slope; how to best weigh their relative contributions to the observed power spectrum?

Lastly, the findings in this paper and paper I cast a new light on the well known excess correlations seen in pencil beam surveys [35, 36]. Could these be the result of enhanced correlations due to magnification bias, particularly if baryon oscillations are taken into account (see [37])? Could the peculiar features seen in the power spectrum analysis [36] be due in part to the corresponding multiplicative windows $G$ and $\left|\tilde{W}_{\|}\right|^{2}$ ? We hope to address these questions in the future.

\section{Acknowledgments}

We thank Roman Scoccimarro for useful discussions. LH thanks Ming-Chung Chu and the Institute of Theoretical Physics at the Chinese University of Hong Kong for hospitality where part of this work was done. Research for this work is supported by the DOE, grant DE-FG02-92-ER40699, and the Initiatives in Science and Engineering Program at Columbia University. EG acknowledges support from Spanish Ministerio de Ciencia y Tecnologia (MEC), project AYA2006-06341 with ECFEDER funding, and research project 2005SGR00728 from Generalitat de Catalunya.

\section{APPENDIX A: INCORPORATING THE ALCOCK-PACZYNSKI EFFECT}

In this Appendix, we incorporate the Alcock-Paczynski distortion of the correlation function and power spectrum [32, 33]. The observed redshifts and angles are converted to comoving radial and transverse distances using the Hubble parameter and angular diameter distance. Let us denote the parameters used in such a conversion by $\bar{H}^{\mathrm{AP}}$ and $\bar{\chi}^{\mathrm{AP}}$. Suppose the true values for these parameters are $\bar{H}$ and $\bar{\chi}$. Then, the two-point correlation function, taking into account possible Alcock-Paczynski distortion, 
is related to the true two-point correlation by

$$
\xi_{\text {obs }}^{\mathrm{AP}}\left(\mathbf{x}_{\mathbf{1}}{ }^{\mathrm{AP}} ; \mathbf{x}_{\mathbf{2}}{ }^{\mathrm{AP}}\right)=\xi_{\text {obs }}\left(\mathbf{x}_{\mathbf{1}} ; \mathbf{x}_{\mathbf{2}}\right)
$$

where the right hand side is the true two-point correlation given in eq. (29), and $\delta \chi^{\mathrm{AP}}=\delta \chi \bar{H} / \bar{H}^{\mathrm{AP}}$ and $\delta \mathbf{x}_{\perp}^{\mathrm{AP}}=$ $\delta \mathbf{x}_{\perp} \bar{\chi}^{\mathrm{AP}} / \bar{\chi}$

Ignoring for the moment redshift distortion due to peculiar motions, putting eq. (10) into eq. (A1), we have

$$
\begin{aligned}
& \xi_{\mathrm{obs}}^{\mathrm{AP}}\left(\delta \chi^{\mathrm{AP}}, \delta x_{\perp}^{\mathrm{AP}}\right)= \\
& \quad \xi_{g g}\left(\sqrt{\delta \chi^{\mathrm{AP}^{2}}\left(1-\epsilon_{H}\right)^{2}+\delta x_{\perp}^{\mathrm{AP}}\left(1-\epsilon_{\chi}\right)^{2}}\right) \\
& \quad+\left(1-\epsilon_{H}\right) \delta \chi^{\mathrm{AP}} f\left(\left(1-\epsilon_{\chi}\right) \delta x_{\perp}^{\mathrm{AP}}\right)+g\left(\left(1-\epsilon_{\chi}\right) \delta x_{\perp}^{\mathrm{AP}}\right)
\end{aligned}
$$

where we have defined

$$
1-\epsilon_{H} \equiv \bar{H}^{\mathrm{AP}} / \bar{H} \quad, \quad 1-\epsilon_{\chi} \equiv \bar{\chi} / \bar{\chi}^{\mathrm{AP}}
$$

The important point is this: the exercise of separating the three contributions to the observed galaxy correlation, outlined after eq. (10) (see also Fig. 2 in paper I), still works, with minor modifications. For a fixed $\delta x_{\perp}^{\mathrm{AP}}$, the magnification bias corrections $\left(1-\epsilon_{H}\right) \delta \chi^{\mathrm{AP}} f+g$ still dominate over $\xi_{g g}$ in the limit of a large $\delta \chi^{\mathrm{AP}}$. This allows one to fit for the slope $\left(1-\epsilon_{H}\right) f$ and the intercept $g$. The cosmology dependent factor of $1-\epsilon_{H}$ can be absorbed into the galaxy bias factor that is present in $f$, which describes the galaxy-magnification correlation. The extrapolation back to $\delta \chi^{\mathrm{AP}}=0$ gives the intercept $g$ which describes the magnification-magnification correlation. Both $f$ and $g$ are determined up to an overall rescaling of their argument $\delta x_{\perp}$, which is an uncertainty that is always present to the extent the cosmology dependent angular diameter distance is uncertain. $\xi_{g g}$ can be obtained by subtracting the inferred galaxy-magnification and magnification-magnification contributions from $\xi_{\mathrm{obs}}^{\mathrm{AP}}$.

It is common to consider the monopole (eq. 16 in paper I). Assuming $\epsilon_{H}, \epsilon_{\chi} \ll 1$, it can be shown that

$$
\begin{aligned}
& \text { monopole of } \xi_{\text {obs }}^{\mathrm{AP}}\left(\delta x^{\mathrm{AP}}\right)= \\
& \quad \xi_{g g}\left(\delta x^{\mathrm{AP}}\left(1-\left[\epsilon_{H}+2 \epsilon_{\chi}\right] / 3\right)\right) \\
& \quad+\left(1-\epsilon_{H}\right) \delta x^{\mathrm{AP}} \tilde{f}\left(\delta x^{\mathrm{AP}}\left(1-\epsilon_{\chi}\right)\right)+\tilde{g}\left(\delta x^{\mathrm{AP}}\left(1-\epsilon_{\chi}\right)\right)
\end{aligned}
$$

where

$$
\begin{array}{r}
\tilde{f}\left(\delta x^{\mathrm{AP}}\right) \equiv \int_{0}^{\pi / 2} f\left(\delta x^{\mathrm{AP}} \sin \theta_{x}\right) \cos \theta_{x} \sin \theta_{x} d \theta_{x} \\
\tilde{g}\left(\delta x^{\mathrm{AP}}\right) \equiv \int_{0}^{\pi / 2} g\left(\delta x^{\mathrm{AP}} \sin \theta_{x}\right) \sin \theta_{x} d \theta_{x}
\end{array}
$$

and $\theta_{x}$ is the angle between the separation vector and the LOS.

The appearance of the factor $1-\left[\epsilon_{H}+2 \epsilon_{\chi}\right] / 3$ in the argument of $\xi_{g g}$ is the origin of the common statement that the baryon oscillation scale measures the combination $\left(\bar{\chi}^{2} / \bar{H}\right)^{1 / 3}$ [14], if one examines the monopole.
Note, however, the presence of the anisotropic corrections introduced by magnification bias implies the observed monopole is no longer related to the true monopole by this overall rescaling of $\delta x$.

Let us give the expression for the observed correlation function in the presence of both peculiar motions and the Alcock-Paczynski effect:

$$
\begin{aligned}
& \xi_{\mathrm{obs}}^{\mathrm{AP}}\left(\delta \mathbf{x}^{\mathrm{AP}}\right)= \\
& \quad \int \frac{d^{3} k}{(2 \pi)^{3}} P_{g g}(\bar{z}, k)\left(1+\frac{f_{D}}{b} \frac{k_{\|}^{2}}{k^{2}}\right)^{2} e^{-k_{\|}^{2} \sigma_{z}^{2}} e^{i \mathbf{k} \cdot \delta \mathbf{x}} \\
& +q b(1+\bar{z}) \int \frac{d^{2} k_{\perp}}{(2 \pi)^{2}} P_{g m}\left(\bar{z}, k_{\perp}\right) e^{i \mathbf{k}_{\perp} \cdot \delta \mathbf{x}_{\perp}} \\
& \quad \int \frac{d \chi_{1}^{\prime} d \chi_{2}^{\prime}}{2 \pi \sigma_{z}^{2}} e^{-\frac{\left(\chi_{1}-\chi_{1}^{\prime}\right)^{2}}{2 \sigma_{z}^{2}}} e^{-\frac{\left(\chi_{2}-\chi_{2}^{\prime}\right)^{2}}{2 \sigma_{z}^{2}}}\left|\chi_{1}^{\prime}-\chi_{2}^{\prime}\right| \\
& +(q b)^{2} \int_{0}^{\bar{\chi}} d \chi^{\prime}\left[\frac{\left(\bar{\chi}-\chi^{\prime}\right) \chi^{\prime}}{\bar{\chi}}\right]^{2}\left(1+z^{\prime}\right)^{2} \\
& \quad \int \frac{d^{2} k_{\perp}}{(2 \pi)^{2}} P_{m m}\left(z^{\prime}, k_{\perp}\right) e^{i \mathbf{k}_{\perp} \cdot \delta \mathbf{x}_{\perp}}
\end{aligned}
$$

where $f_{D}$ and $q$ are as defined in eq. (37) and (38), and $\delta \mathbf{x}^{\mathrm{AP}}$ and $\delta \mathbf{x}$ are related as in eq. (A1). The first term on the right accounts for both the Kaiser effect and, in a crude form, the finger-of-god effect due to either virialized motions or photometric redshifts. The second term on the right represents the contribution from galaxy-magnification correlation. The finger-of-god dispersion has an effect here, but its effect is negligible for large LOS separations $\left(\left|\chi_{1}-\chi_{2}\right| \gg \sigma_{z}\right)$ where the galaxymagnification correlation has the largest effect (i.e. the integral over $\chi_{1}^{\prime}$ and $\chi_{2}^{\prime}$ yields roughly $\left.\left|\chi_{1}-\chi_{2}\right|=|\delta \chi|\right)$. The third term on the right is exactly $\xi_{\mu \mu}$ in eq. (8): the finger-of-god dispersion has no effect on this term, because it has no intrinsic dependence on the LOS separation $\delta \chi$. In other words, to good approximation (unless $\sigma_{z}$ is very large), the galaxy-magnification and magnification-magnification terms take the same form as in eq. A2 . This means the exercise of separating the three different contributions to the observed correlation can be repeated here as well.

Lastly, let us give the corresponding expression in Fourier space. Defining $\mathbf{k}^{\mathrm{AP}}$, which is related to $\mathbf{k}$ by

$$
k_{\|}^{\mathrm{AP}}=k_{\|} \bar{H}^{\mathrm{AP}} / \bar{H} \quad, \quad \mathbf{k}_{\perp}{ }^{\mathrm{AP}}=\mathbf{k}_{\perp} \bar{\chi} / \bar{\chi}^{\mathrm{AP}},
$$

the Alcock-Paczynski distorted power spectrum $P_{\mathrm{obs}}^{\mathrm{AP}}$ is related to the true (windowed) power spectrum $P_{\mathrm{obs}}$ by

$$
P_{\mathrm{obs}}^{\mathrm{AP}}\left(\mathbf{k}^{\mathrm{AP}}\right)=\frac{\bar{H}}{\bar{H}^{\mathrm{AP}}} \frac{\left(\bar{\chi}^{\mathrm{AP}}\right)^{2}}{\bar{\chi}^{2}} P_{\mathrm{obs}}(\mathbf{k})
$$

where $P_{\text {obs }}$ is given by

$$
\begin{aligned}
& P_{\mathrm{obs}}(\mathbf{k})= \\
& \qquad \frac{d^{3} k^{\prime}}{(2 \pi)^{3}} P_{g g}\left(\bar{z}, k^{\prime}\right)\left(1+\frac{f_{D}}{b} \frac{\left(k_{\|}^{\prime}\right)^{2}}{{k^{\prime}}^{2}}\right)^{2}
\end{aligned}
$$




$$
\begin{aligned}
& e^{-k_{\|}^{\prime 2} \sigma_{z}^{2}}\left|\tilde{W}\left(\mathbf{k}-\mathbf{k}^{\prime}\right)\right|^{2} \\
+ & q b(1+\bar{z}) G_{z}\left(k_{\|}\right) \int \frac{d^{2} k_{\perp}^{\prime}}{(2 \pi)^{2}} P_{g m}\left(\bar{z}, k_{\perp}^{\prime}\right) \\
& \left|\tilde{W}_{\perp}\left(\mathbf{k}_{\perp}-\mathbf{k}_{\perp}^{\prime}\right)\right|^{2} \\
+ & (q b)^{2}\left|\tilde{W}_{\|}\left(k_{\|}\right)\right|^{2} \int_{0}^{\bar{\chi}} d \chi^{\prime}\left[\frac{\left(\bar{\chi}-\chi^{\prime}\right) \chi^{\prime}}{\bar{\chi}}\right]^{2}\left(1+z^{\prime}\right)^{2} \\
& \int \frac{d^{2} k_{\perp}^{\prime}}{(2 \pi)^{2}} P_{m m}\left(z^{\prime}, k_{\perp}^{\prime}\right)\left|\tilde{W}_{\perp}\left(\mathbf{k}_{\perp}-\mathbf{k}_{\perp}^{\prime} \chi^{\prime} / \bar{\chi}\right)\right|^{2}
\end{aligned}
$$

It is instructive to integrate out the convolving windows following eq. (35), and obtain:

$$
\begin{aligned}
& P_{\mathrm{obs}}^{\mathrm{AP}}\left(\mathbf{k}^{\mathrm{AP}}\right)=\left(1+\epsilon_{H}\right)\left(1+\epsilon_{\chi}\right)^{2} P_{g g}(\bar{z}, k) \\
& {\left[\left(1+\frac{f_{D}}{b} \frac{k_{\|}^{2}}{k^{2}}\right)^{2} e^{-k_{\|}^{2} \sigma_{z}^{2}}+\right.} \\
& q(1+\bar{z}) G\left(k_{\|}\right) \frac{P_{m m}\left(\bar{z}, k_{\perp}\right)}{P_{m m}(\bar{z}, k)}+
\end{aligned}
$$

$$
\left.q^{2}\left|\tilde{W}_{\|}\left(k_{\|}\right)\right|^{2} \int_{0}^{\bar{\chi}} d \chi^{\prime}\left(\bar{\chi}-\chi^{\prime}\right)^{2}\left(1+z^{\prime}\right)^{2} \frac{P_{m m}\left(z^{\prime}, k_{\perp} \bar{\chi} / \chi^{\prime}\right)}{P_{m m}(\bar{z}, k)}\right]
$$

where we have adopted the approximation $G_{z} \sim G$ as is done in eq. (36). Note how the distinctive multiplicative windows $G$ and $\left|\tilde{W}_{\|}\left(k_{\|}\right)\right|^{2}$, which are signatures of the galaxy-magnification and magnificationmagnification correlations, remain intact in the presence of the Alcock-Paczynski effect. They give rise to an enhancement in the observed correlation that is localized to low $k_{\|}$'s. Just as in the case of the real space correlation function, quantities such as $P_{g m}$ and $P_{m m}$ (which are related to Fourier transforms of $f$ and $g$ in eq. A2 ) can be determined from data only up to a rescaling of their argument $k_{\perp}$ (eq. [A7]) (and in the case of $P_{g m}$, an overall normalization which can be absorbed into the galaxy bias).
[1] L. Hui, E. Gaztanaga, M. LoVerde, submitted to Phys. Rev. D (2007) arXiv:0706.1071 (paper I).

[2] T. Matsubara, Astrophys. J. Lett. 537, 77 (2000).

[3] E. L. Turner, J. P. Ostriker, J. R. Gott, Astrophys. J. 284, 1 (1984); R. L. Webster,P. C. Hewett, M. E. Harding, G. A. Wegner, Nature 336, 358 (1988); W. Fugmann, Astron. \& Astrophys. 204, 73 (1988); R. Narayan, Astrophys. J. Lett. 339, 53 (1989); P. Schneider, Astron. \& Astrophys. 221, 221 (1989).

[4] J. Villumsen, preprint (1995) astro-ph/9512001.

[5] T. J. Broadhurst, A. N. Taylor, J. A. Peacock, Astrophys. J. 438, 49 (1996).

[6] J. Villumsen, W. Freudling, L. N. da Costa, Astrophys. J. 481, 578 (1997).

[7] R. Moessner, B. Jain, J. Villumsen, Mon. Not. R. Astron. Soc. 294, 291 (1998)

[8] R. Moessner, B. Jain, Mon. Not. R. Astron. Soc. 294, L18 (1998).

[9] E. Gaztañaga, Astrophys. J. 589, 82 (2003).

[10] R. Scranton, et al. , Astrophys. J. 633, 589 (2005).

[11] B. Menard, . Bartelmann, Astron. \& Astrophys. 386, 784 (2002).

[12] B. Jain, Astrophys. J. Lett. 580, 3 (2002).

[13] B. Jain, R. Scranton, R. K. Sheth, Mon. Not. R. Astron. Soc. 345, 62 (2003).

[14] D. J. Eisenstein et al. , Astrophys. J. 633, 560 (2005)

[15] S. Cole et al. , Mon. Not. R. Astron. Soc. 362, 505 (2005)

[16] W. J. Percival et al. , preprint (2006) astro-ph/0608635

[17] G. Hütsi, Astron. \& Astrophys. 449, 891 (2006)

[18] M. Tegmark et al. , preprint (2006) astro-ph/0608632

[19] An incomplete list of theoretical papers on the use of baryon oscillations to measure dark energy includes: C. Blake, K. Glazebrook, Astrophys. J. 594, 665 (2003); E. Linder, Phys. Rev. D 68, 082504 (2003); W. Hu, Z. Haiman, Phys. Rev. D 68, 063004 (2003); J. J. Seo, D. J. Eisenstein, Astrophys. J. 598, 720 (2003); A. Cooray, Mon. Not. R. Astron. Soc. 348, 250 (2004); T. Matsubara, Astrophys. J. 615, 573 (2004); L. Amendola,
C. Quercellini, \& E. Giallongo, Mon. Not. R. Astron. Soc. 357, 429 (2005); C. Blake \& S. Bridle, Mon. Not. R. Astron. Soc. 363, 1329 (2005).

[20] An incomplete list of planned/proposed surveys to measure the baryon acoustic oscillations includes: ADEPT (http://www.jhu.edu/news_info/news/home06/aug06/ adept.html), DES Dark Energy Survey (Annis et al. , from http://decam.fnal.gov, http://www.darkenergysurvey.org), FMOS/WFMOS at Gemini/Subaru astro-ph/0507457, HETDEX (http://www.as.utexas.edu/hetdex), WiggleZ (http://astronomy.swin.edu.au/ karl/KarlHome/Home.html).

[21] A. Vallinotto, S. Dodelson, C. Schmid, J.-P. Uzan (2007) astro-ph/0702606.

[22] M. LoVerde, L. Hui, E. Gaztañaga, submitted to Phys. Rev. D (2007) arXiv:0708.0031.

[23] C. Wagner, V. Muller, M. Steinmetz, preprint (2007) arXiv:0705.0354.

[24] P. Zhang, X. Chen, preprint (2007) arXiv:0710.1486.

[25] M. LoVerde, L. Hui, E. Gaztañaga, Phys. Rev. D 75, 043519 (2007) astro-ph/0611539

[26] D. J. Eisenstein, W. Hu, Astrophys. J. 496, 605 (1998)

[27] R. E. Smith et al. , Mon. Not. R. Astron. Soc. 341, 1311 (2003)

[28] A. Cooray, W. Hu, D. Huterer, M. Joffre, Astrophys. J. Lett. 557, 7 (2001)

[29] N. Kaiser, Astrophys. J. 498, 26 (1998)

[30] R. Scoccimarro, Phys. Rev. D 70, 083007 (2004)

[31] A. Hamilton, Proceedings of the Ringberg Workshop on Large Scale Structure, Edited by D. Hamilton, Kluwer Academic, Dordrecht (1996) astro-ph/9708102

[32] C. Alcock, B. Paczynski, Nature 281, 358 (1979)

[33] T. Matsubara, Y. Suto, Astrophys. J. Lett. 470, 1 (1996); T. Matsubara, A. S. Szalay, Phys. Rev. Lett. 90, 21302 (2003)

[34] R. E. Smith, R. Scoccimarro, R. K. Sheth, preprint (2006) astro-ph/0609547 and (2007) astro-ph/0703620 
[35] T. J. Broadhurst, R. S. Ellis, D. C. Koo, A. S. Szalay, Nature 343, 726 (1990).

[36] A. Szalay, T. J. Broadhurst, N. Ellman, D. C. Koo, R. S. Ellis, Proc. Natl. Acad. Sci. 90, 4853 (1993).
[37] D. J. Eisenstein, W. Hu, J.Silk \& A.Szalay Astrophys. J. Lett. 494, L1 (1998) 